\title{
MORBIDADE HOSPITALAR EM IDOSOS DO PARANÁ DURANTE O ANO DE
} 2016

\section{Mateus Dias Antunes ${ }^{1}$; Fernanda Shizue Nishida ${ }^{2}$}

${ }^{1}$ Fisioterapeuta, Mestre em Promoção da Saúde pelo Centro Universitário de Maringá - UNICESUMAR; Bolsista da Coordenação de Aperfeiçoamento de Pessoal de Nível Superior (CAPES) - Maringá, Paraná, Brasil.

${ }^{2}$ Enfermeira. Doutora em Ciências - Universidade de São Paulo, docente no Programa de Mestrado em Promoção da Saúde do Centro Universitário de Maringá

- UNICESUMAR; Pesquisadora do Instituto Cesumar de Ciência, Tecnologia e Inovação (ICETI) - Maringá, Paraná, Brasil. fernanda_nishida@hotmail.com

Recebido em: 02/10/2017 - Aprovado em: 21/11/2017 - Publicado em: 05/12/2017 DOI: 10.18677/EnciBio_2017B99

\begin{abstract}
A presença de múltiplas morbidades na população idosa é decorrente dos efeitos acumulados às exposições à estressores durante os ciclos da vida. O objetivo foi caracterizar as cinco principais causas de morbidade hospitalar em idosos atendidos no Sistema Único de Saúde em 2016 no Paraná. Delineamento transversal que utilizou dados obtidos do SIH/SUS do DataSus. Foram incluídos todos os 169.274 registros de morbidade hospitalar em idosos residentes no Paraná, segundo capítulo do CID-10 em 2016. Dentre as causas de morbidade hospitalar de idosos no SUS, verificou-se que entre as principais, a causa primordial é decorrente de doenças do aparelho circulatório (36,85\%), seguido de doenças do aparelho respiratório. Concluiu-se que as doenças do aparelho circulatório são as principais morbidades hospitalares de idosos no Paraná, sua relevância mostra a necessidade de intensificar as ações de saúde para a redução, controle, proteção e promoção da saúde através da melhoria na qualidade de vida dos idosos.
\end{abstract}

RESUMO

PALAVRAS-CHAVE: Envelhecimento, Epidemiologia, Inquéritos de Morbidade, Promoção da Saúde.

\section{HOSPITAL MORBIDITY IN ELDERLY PARANÁ DURING THE YEAR 2016}

\begin{abstract}
The presence of multiple morbidities in the elderly population is due to the cumulative effects of exposure to stressors during life cycles. The objective was to characterize the five main causes of hospital morbidity in the elderly attended in the Unified Health System in 2016 in Paraná. Transverse design using data obtained from the SIHS / SUS DataSus. We included all 169,274 hospital morbidity records in elderly people living in Paraná, according to the ICD-10 chapter in 2016. Among the causes of hospital morbidity of the elderly in SUS, it was verified that among the main ones, the primary cause is due to diseases of the circulatory system $(36.85 \%)$, followed by diseases of the respiratory system. It was concluded that diseases of the circulatory system are the main hospital morbidities of the elderly in Paraná, its relevance shows ENCICLOPÉDIA BIOSFERA, Centro Científico Conhecer - Goiânia, v.14 n.26; p. 1166 2017
\end{abstract}


the need to intensify health actions for the reduction, control, protection and health promotion through the improvement in the quality of life of the elderly .

KEYWORDS: Aging, Epidemiology, Morbidity Surveys, Health Promotion.

\section{INTRODUÇÃO}

Ao longo das últimas décadas, tem se destacado um rápido crescimento populacional em diversos países. No Brasil, a população idosa é a que mais cresce, em 2013 este grupo representava 12,5\% da população total (AMORIM et al., 2017), e 2016, esse contingente atingiu os 30\% (FERREIRA et al., 2017).

No Paraná, o acelerado aumento dos idosos comparado aos jovens e adultos merece destaque. No ano de 2010, este grupo correspondia a $11,2 \%$ da população paranaense total. Esta situação acarreta alterações nos padrões econômicos e sociais do estado, visto que estas populações apresentam um aumento da demanda por serviços de saúde específicos e consequentemente mais custos (KERNKAMP et al., 2016).

Paralelo a este fenômeno surge à necessidade de maior uso dos serviços de saúde em consequência do perfil epidemiológico deste grupo, caracterizado por declínio funcional, doenças crônicas e aumento de morbidades. Esta população apresenta alta taxa de morbidade hospitalar, levando a recorrentes internações, bem como, maior permanência nos leitos hospitalares (AMORIM et al., 2017). A alta prevalência das doenças crônicas e a presença de múltiplas morbidades nesta população são decorrentes dos efeitos acumulados às exposições a estressores durante os ciclos da vida. Conhecer quais são as morbidades reforça a estratégia de orientar as ações e estratégias em saúde para os idosos (BORIM et al., 2017).

Vem se ampliando no Brasil o interesse em utilizar banco de dados originados, de forma rotineira, pelos serviços de saúde, como ferramenta no planejamento, avaliação e manutenção de políticas públicas e serviços em saúde (BITTENCOURT et al., 2006). Diante disso, o Sistema de Informações Hospitalares do Sistema Único de Saúde (SIH/SUS) é um banco de dados desenhado para fins administrativos, usados através de um sistema que é responsável pelo processamento dos registros contidos nos formulários de Autorização de Internação Hospitalar (AIH) (AMARAL et al., 2004).

São escassos, estudos atuais com os dados das principais morbidades hospitalares no Paraná. Nesse sentido, este estudo teve por objetivo caracterizar as cinco principais causas de morbidade hospitalar em idosos atendidos no Sistema Único de Saúde no ano de 2016 no Paraná.

\section{MATERIAL E MÉTODOS}

Foi realizado um estudo de delineamento transversal, de caráter exploratório. Os dados foram obtidos do SIH/SUS do Datasus. Foram incluídos todos os 169.274 registros em 2016 de morbidade hospitalar em idosos residentes no Paraná, por meio de capítulo do CID-10.

No presente estudo, foi dispensada a apreciação pelo Comitê de Ética em Pesquisa (CEP) visto que se tratam de informações públicas, disponíveis on-line. Segundo o Conselho Nacional de Saúde (CNS) e a Comissão Nacional de Ética em Pesquisa (CONEP) estudos envolvendo apenas dados de domínio público onde não 
seja possível a identificação dos indivíduos da pesquisa não requerem aprovação do Sistema CEP-CONEP (http://conselho.saude.gov.br/).

Foram coletados registros do mês de janeiro a dezembro de 2016 . Este levantamento foi realizado por dois pesquisadores no dia dois de setembro de 2017. Os dados foram tabulados no Microsoft Excel 2010 e apresentados por meio da estatística descritiva (frequência absoluta e relativa, gráficos e tabelas).

\section{RESULTADOS E DISCUSSÃO}

No período estudado, foram identificados 169.274 registros. Deste total $74,58 \%(\mathrm{n}=126.237)$ são indivíduos da cor branca, 2,04\% ( $\mathrm{n}=3448)$ negra, 9,55\% $(\mathrm{n}=16164)$ parda, e $0,75 \%(\mathrm{n}=1277)$ indígena, dados não informados representaram $13,05 \%(n=22093)$ das notificações para esta variável.

Dentre as causas de morbidade hospitalar de idosos no SUS, a causa primordial é decorrente de doenças do aparelho circulatório $(36,85 \%)$, seguido de doenças do aparelho respiratório. Em último verificam-se internações por causas externas/lesões por envenenamento $(10,69 \%)$, categoria em que se enquadram internações por atropelamento, quedas, violência, entre outros (Figura 1).

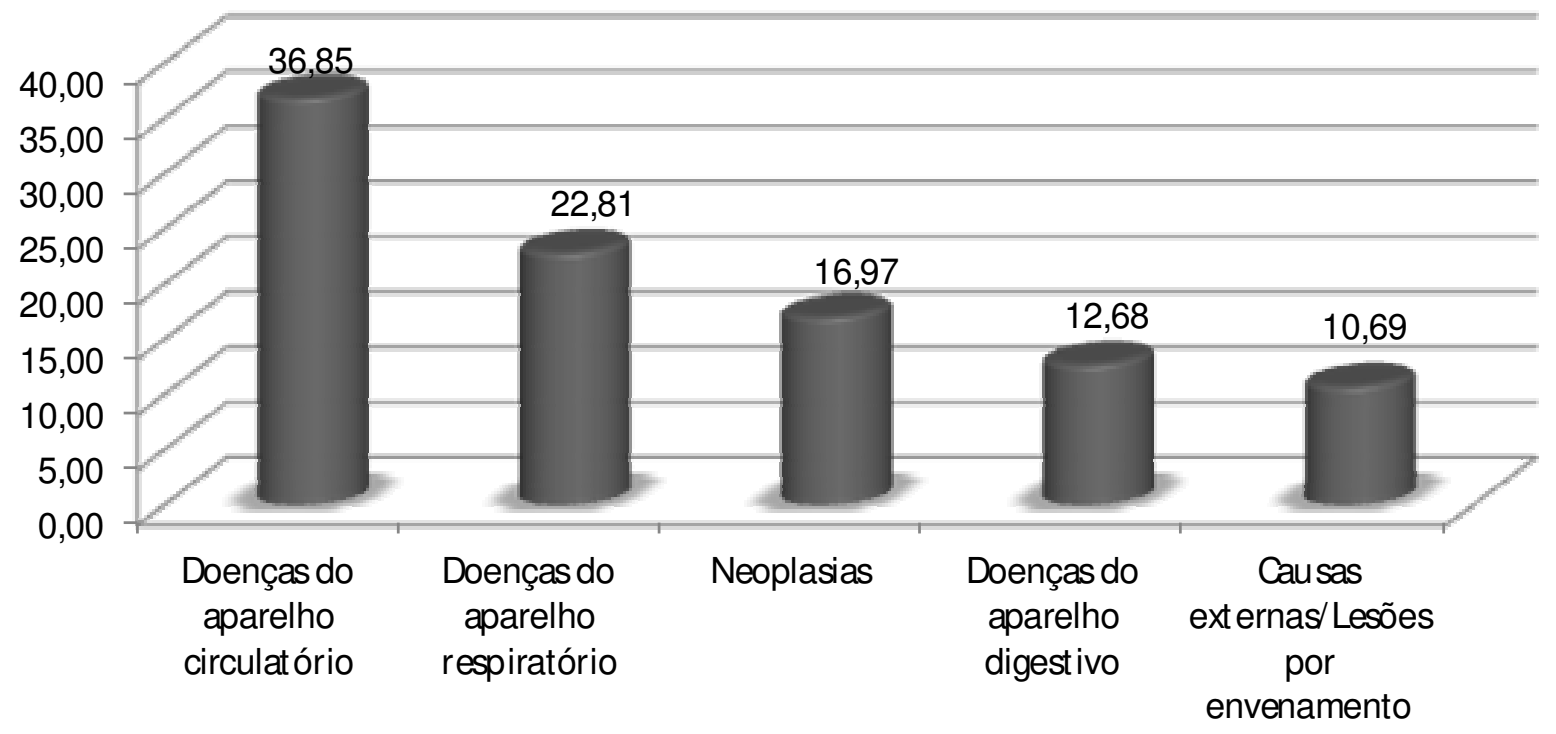

FIGURA 1. Registros hospitalares de morbidade em idosos no Paraná, 2016. SIHSUS, (2017).

Marques et al. (2014) observaram que as doenças do aparelho circulatório são as maiores responsáveis pelas internações hospitalares em idosos. Do mesmo modo, estudo conduzido por Castro et al., (2013) relataram que no Paraná, as internações de idosos mais prevalentes foram doenças do aparelho circulatório $(28,7 \%)$ e respiratório $(21,3)$, seguida por neoplasias $(8,8 \%)$ e doenças do aparelho digestivo (8,7\%). Achados semelhantes ao observado neste estudo, embora 0 percentual de doenças do aparelho circulatório e neoplasias tenham tido um aumento expressivo. Esses achados reforçam a importância desse sistema de informação em saúde como fonte para monitoramento e planejamento das ações de promoção da saúde voltadas à população idosa no Paraná. 
Verificou-se que $43,16 \%$ (73063) dos casos de morbidade ocorreram com idosos na faixa etária dos $60-69$ anos de idade, 35,37\% (59873) idade entre $70-79$ anos e 21,47\% (36338) acima de 80 anos. Estudos semelhantes com idosos verificaram que a maioria das internações ocorre na faixa etária de 60 a 69 anos (CASTRO et al., 2013; KERNKAMP et al., 2016). Estudo que buscou realizar um diagnóstico da população idosa brasileira verificou que as taxas de mortalidade por doenças do aparelho circulatório aumentam com a idade em ambos os sexos, porém essas taxas sofreram um declínio entre 1980 a 1996 e aumentando nas faixas etárias mais longevas (acima de 70 anos) (COSTA et al., 2000).

Após as causas de morbidade por doenças do aparelho circulatório, neoplasias se constituem o segundo fator de internação entre idosos de 60-69 anos. Causas externas/lesões por envenenamento acometem com mais frequência idosos com idade superior a 80 anos do que doenças do aparelho digestivo (Figura 2).

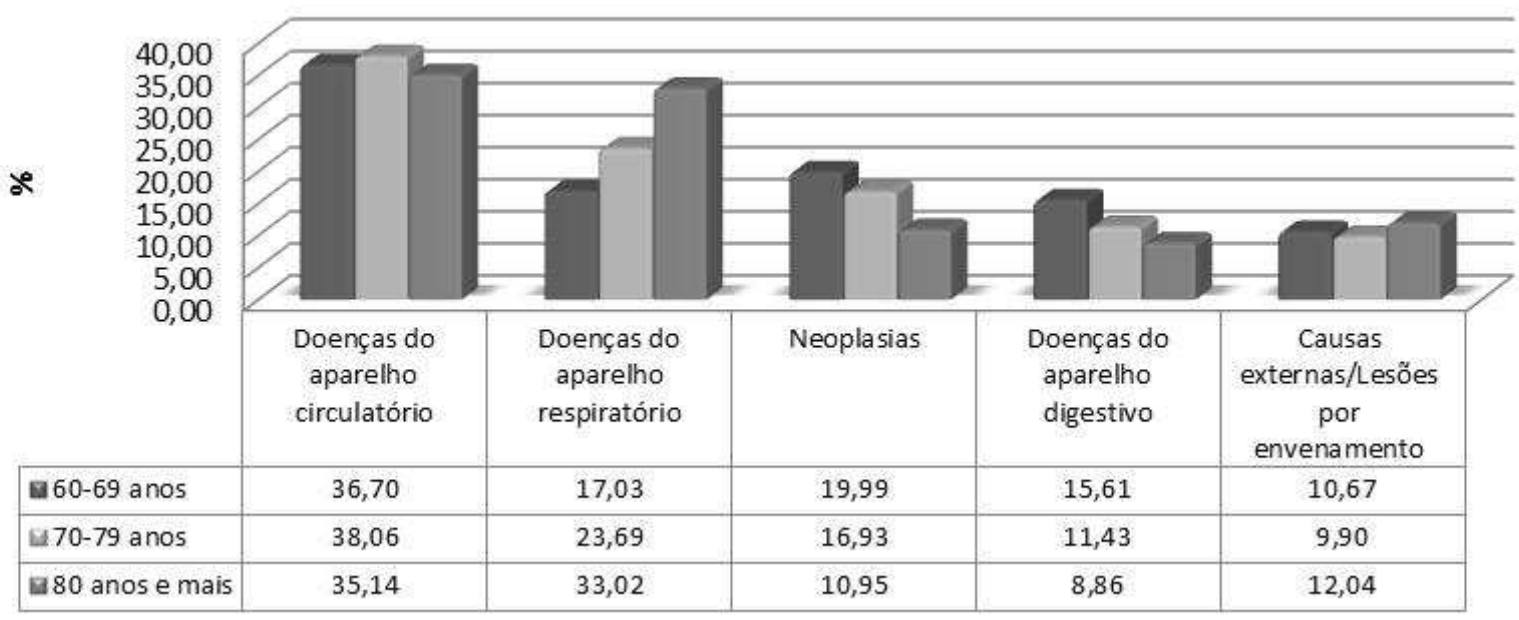

FIGURA 2. Registros hospitalares de morbidade das cinco principais causas em idosos no Paraná, por faixa de idade, 2016. SIH-SUS, (2017).

Estudo que analisou idosos com neoplasias, verificou que as demandas por internação hospitalar no período de 2010 à 2012 e 2010 à 2014, porque foram identificados aumentos ainda mais significativos, de $16,15 \%$ em dois anos e $24,69 \%$ em quatro anos. Nesse sentido, apresenta um quadro de realidade sociodemográfica e de saúde brasileira, que necessita de demandas multidimensionais de cuidados em saúde e prioridades de estudos (SILVA et al., 2016).

Notou-se que as neoplasias eram consideradas antigamente como a segunda causa de morte de idosos brasileiros, bem como nos Estados Unidos até o ano de 1996 (COSTA et al., 2000). As doenças do aparelho respiratório subiram para o segundo lugar entre as causas de morbidade, como também foi possível verificar neste estudo. Atualmente, essas doenças são bastante comuns e as formas de contraí-las são muito abrangentes. À vista disso, são necessários estudos para identificar os períodos de maior ocorrência de internações para identificar os fatores que aumentam essa morbidade (ANTUNES et al., 2017).

Referente ao tempo de internação, foi observado que em 2016 foram 913.557 dias de internação para pessoas do sexo masculino e 745.869 dias para o sexo feminino. A média de dias de internação foi maior para as doenças do aparelho ENCICLOPÉDIA BIOSFERA, Centro Científico Conhecer - Goiânia, v.14 n.26; p. 1169 
circulatório nos homens, 4,7 dias, assim como para as mulheres com 4,3 dias (Figura 3).

\begin{tabular}{|c|c|c|c|c|c|c|}
\hline \multirow[t]{2}{*}{ Capítulo CID-10 } & \multicolumn{2}{|c|}{ Masculino } & \multicolumn{2}{|c|}{ Feminino } & \multicolumn{2}{|c|}{ Total } \\
\hline & $\begin{array}{c}\text { Dias de } \\
\text { parmanência }\end{array}$ & Média de dias & $\begin{array}{c}\text { Dias de } \\
\text { parmanência }\end{array}$ & Média de dias & $\begin{array}{c}\text { Dias de } \\
\text { parmanência }\end{array}$ & Média de dias \\
\hline Doenças do aparelho circulatório & 248.676 & 4,7 & 214.167 & 4,3 & 462.843 & 4,5 \\
\hline Doenças do aparelho respiratório & 223.255 & 4,4 & 205.293 & 4,2 & 428.548 & 4,3 \\
\hline Neoplasias & 114.991 & 3,9 & 113.970 & 3,2 & 228.961 & 3,5 \\
\hline Doenças do aparelho digestivo & 125.962 & 3,2 & 114.784 & 2,9 & 240.746 & 3,1 \\
\hline Causas externas/Lesões por envenamento & 200.673 & 3,5 & 97.655 & 3,5 & 298.328 & 3,5 \\
\hline Total & 913.557 & & 745.869 & & 1.659 .426 & \\
\hline
\end{tabular}

FIGURA 3. Registros hospitalares de morbidade das cinco principais causas em idosos no Paraná, por tempo de internação, 2016.

Fonte: SIH-SUS, ( 2017)

Em um contexto geral, a média de dias de internação de indivíduos do sexo masculino foi maior do que no feminino. Este estudo teve média de dias de internação inferior ao estudo realizado com idosos de Goiânia no ano de 2009, onde foi verificado tempo médio das hospitalizações de 6,2 dias (PAGOTTO et al. 2013). Referente ao sexo, estudo realizado em São Paulo e Ribeirão Preto verificaram-se que mulheres buscaram os serviços de saúde quase duas vezes mais em relação aos homens (LEVORATO et al., 2014). É possível que a maior procura da população feminina pelos serviços de saúde, tenha algum efeito na manutenção da saúde das mulheres, o que poderia reduzir os dias de internação hospitalar.

Foi analisado o valor total gasto com as AlH de acordo com o sexo. Foi possível verificar que o montante de recursos gastos com doenças do aparelho circulatório é quase o dobro do valor gasto com neoplasias nas mulheres e praticamente o triplo entre os homens. Nos homens, o segundo maior gasto é decorrente de internações por causas externas/lesões por envenenamento, já nas mulheres esse é o menor custo gerado dentre as cinco principais causas (Figura 4).

Em 2016 foram gastos $R \$ 757.796 .157,43$ reais com estas cinco causas de morbidade, valor que representa $62 \%$ do total gasto ( $R \$ 1.224 .620 .812,58)$ nas internações hospitalares em 2016. A partir dos resultados, evidencia-se a importância que as doenças do aparelho circulatório, respiratório, bem como as neoplasias devem continuar a receber maior enfoque, uma vez que os gastos destinados as internações e tratamentos geram maior necessidade de recursos por parte do sistema público de saúde (KUMMER et al., 2016). Estes dados são apresentados na figura 4: 


\begin{tabular}{|c|c|c|c|}
\hline \multirow[b]{2}{*}{ Capítulo CID 10} & \multicolumn{2}{|c|}{ Sexo } & \multirow[b]{2}{*}{ Total } \\
\hline & Masculino & Feminino & \\
\hline Doenças do aparelho circulatório & $\mathrm{R} \$ 177.486 .278,62$ & $\mathrm{R} \$ 127.245 .889,97$ & $\mathrm{R} \$ 304.732 .168,59$ \\
\hline Doenças do aparelho respiratório & $R \$ 56.295 .495,08$ & $R \$ 49.349 .940,15$ & $R \$ 105.645 .435,23$ \\
\hline Neoplasias & $\mathrm{R} \$ 63.738 .247,88$ & $\mathrm{R} \$ 71.136 .048,68$ & $\mathrm{R} \$ 134.874 .296,56$ \\
\hline Doenças do aparelho digestivo & $\mathrm{R} \$ 48.125 .624,14$ & $\mathrm{R} \$ 40.227 .615,36$ & $\mathrm{R} \$ 88.353 .239,50$ \\
\hline Causas externas/lesões por envenenamento & $\mathrm{R} \$ 84.802 .262,56$ & $\mathrm{R} \$ 39.388 .754,99$ & $\mathrm{R} \$ 124.191 .017,55$ \\
\hline Total & $\mathrm{R} \$ 430.447 .908,28$ & $\mathrm{R} \$ 327.348 .249,15$ & $\mathrm{R} \$ 757.796 .157,43$ \\
\hline
\end{tabular}

FIGURA 4. Registros hospitalares de morbidade das cinco principais causas em idosos no Paraná por valor total gasto, 2016. SIH-SUS, (2017).

O coeficiente de mortalidade foi de 2,22 a 8,95 óbitos de cada 1000 pessoas, tendo sido maior nas neoplasias com 8,95/1000 óbitos nos homens e 6,37/1000 nas mulheres. A segunda maior taxa de mortalidade foi para doenças do aparelho respiratório. A menor taxa foi devido a causas externas/lesões por envenenamento com 2,22/1000 em ambos os sexos (figura 5).

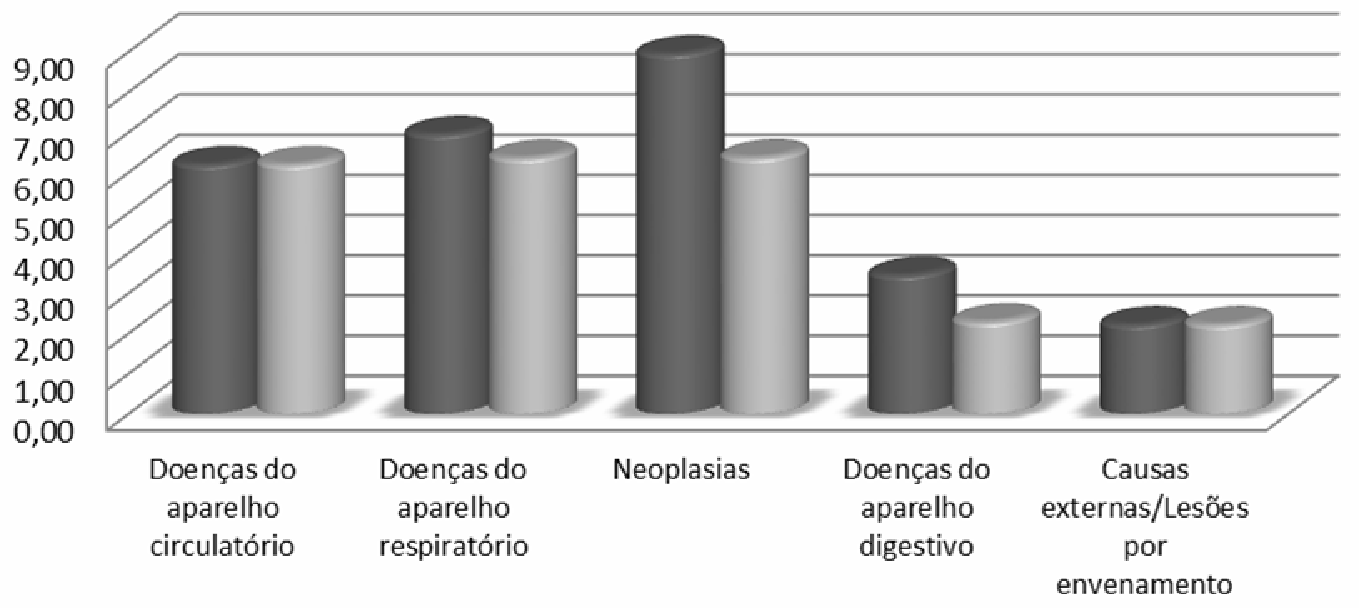

a Masculino $\square$ Feminino

FIGURA 5. Taxa de mortalidade das cinco principais causas em idosos no Paraná por sexo, 2016. SIH-SUS, (2017).

Embora as doenças do aparelho circulatório estejam entre as causas mais frequentes de morte no Brasil (COSTA et al., 2016; BRANT et al., 2017) deve-se considerar que a população vem crescendo e mudanças acompanham esse crescimento, estudos relatam o declínio das taxas de mortalidade decorrentes das doenças cardiovasculares como resultado da mudança no estilo de vida, melhoria de tecnologia para prevenção e promoção da saúde e da implementação de diferentes políticas de saúde pública (MANSUR; FAVARATO, 2012; LUZ et al., 2017). Este declínio foi maior no Brasil nos estados do Sul e Sudeste, em relação aos estados do Norte e Nordeste (BRANT et al., 2017).

As ações focadas na educação em saúde representam a possibilidade de mudanças, partindo-se de perspectivas dialógicas, emancipadoras, participativas e 
criativas, que promovem maior autonomia do idoso, no que diz respeito à sua condição de sujeito de direitos e autor de sua trajetória de saúde e doença (MALLMAN et al., 2015).

\section{CONCLUSÃO}

É possível concluir que as doenças do aparelho circulatório constituem a principal causa de morbidade hospitalar de idosos no Paraná, a grande relevância traz à luz a necessidade de intensificar as ações de saúde para a redução, controle, proteção e promoção da saúde e qualidade de vida dos idosos.

\section{AGRADECIMENTOS}

Ao Centro Universitário de Maringá, à Coordenação de Aperfeiçoamento de Pessoal de Nível Superior (CAPES) e Instituto Cesumar de Ciência, Tecnologia e Inovação - ICETI que contribuíram com esta investigação.

\section{REFERÊNCIAS}

AMARAL, A. C. S.; COELI, C. M.; COSTA, M. D. C. E. D.; CARDOSO, V. D. S.; TOLEDO, A. L. A. D. et al. Morbidity and mortality profile of hospitalized elderly patients. Cadernos de Saúde Pública, v. 20, n. 6, p. 1617-1626, 2004. DOI: http://dx.doi.org/10.1590/S0102-311X2004000600020

AMORIM, D. N. P.; CHIARELLO, M. D.; VIANNA, L. G.; MORAES, C. F.; VILAÇA, K. $\mathrm{H}$. C. Interactions through conditions sensitive to primary attention of elderly persons in Brazil, 2003 to 2012. Journal of Nursing UFPE on line, v. 11, n. 2, p. 576-583, 2017.

http://www.revista.ufpe.br/revistaenfermagem/index.php/revista/article/view/10083

ANTUNES, M. D.; BUENO, G. R. ; BRIDA, R. L. ; VIEIRA, G. C. G. ; NISHIDA, F. S. et al. Evolução das internações e gastos em saúde por Doenças do Aparelho Respiratório em idosos em Maringá - PR. Revista Sodebrás, v. 12, n. 137, p. 93-98, 2017. Disponível em: http://www.sodebras.com.br/edicoes/N137.pdf

BITTENCOURT, S. A.; CAMACHO, L. A. B.; LEAL, M. C. Hospital Information Systems and their application in public health. Cadernos de Saúde Pública, v. 22, n. 1, p. 19-30, 2006. DOI: http://dx.doi.org/10.1590/S0102-311X2006000100003

BORIM, F. S. A.; FRANCISCO, P. M. S. B.; NERI, A. L. Sociodemographic and health factors associated with mortality in community-dwelling elderly. Revista de Saúde Pública, v. 51, p. 42-53, 2017. DOI: http://dx.doi.org/10.1590/s15188787.2017051006708

BRANT, L. C. C.; NASCIMENTO, B. R.; PASSOS, V. M. A.; DUNCAN, B. B.; BENSEÑOR I. J. M. et al. Variations and particularities in cardiovascular disease mortality in Brazil and Brazilian states in 1990 and 2015: estimates from the Global Burden of Disease. Revista Brasileira de Epidemiologia, v. 20, n. 1, p. 116128, 2017. DOI: http://dx.doi.org/10.1590/1980-5497201700050010 
CASTRO, V. C.; BORGHI, A. C.; MARIANO, P. P., FERNANDES, C. A. M, MATHIAS, T. A. F. et al. Perfil de internações hospitalares de idosos no âmbito do Sistema Único de Saúde. Revista da Rede de Enfermagem do Nordeste v. 14, N. 4, p. 791-800, 2013. Disponível em: http://4www.redalyc.org/articulo.oa?id=324028459016

COSTA, F. E.; FROHLICH, C.; GRAPIGLIA, C. Z.; LEITE, H. M.; MORIMOTO, T. Trend analysis of mortality from circulatory diseases in Rio Grande do Sul, 1998 to 2012. Journal of Health \& Biological Sciences, v. 4, n. 2, p. 82-87, 2016. DOI: http://dx.doi.org/10.12662/2317-3076jhbs.v4i2.659.p82-87.2016

COSTA, M. F. F. L.; GUERRA, H. L.; BARRETO, S. M.; GUIMARAES, R. M. Diagnóstico da situação de saúde da população idosa brasileira: um estudo da mortalidade e das internações hospitalares públicas. Informe epidemiológico do SUS, v. 9 , n. 1 , p. $43-50,2000$. DOI: http://dx.doi.org/10.5123/S010416732000000100003

FERREIRA, L. V.; SILVA, M. C. M.; CASTRO, E. A. B.; FRIEDRICH, D. B. C. Busca do autocuidado por idosos na rede de atenção à saúde. Revista Contexto \& Saúde, v. 17, n. 32, p. 46-54, 2017. DOI: http://dx.doi.org/10.21527/2176-7114.2017.32.4654

KERNKAMP, C. D. L.; COSTA, C. K. F.; MASSUDA, E. M.; SILVA, E. S.; YAMAGUCHI, M. U. et al. Morbidity profile and hospital expenses with elderly patients in Paraná State, Brazil, 2008-2012. Cadernos de Saúde Pública, v. 32, n. 7, p. 1-14, 2016. DOI: http://dx.doi.org/10.1590/0102-311X00044115

KUMMER, S. S.; GOMES, I.; STOBÄUS, C. D. Internações hospitalares de idosos residentes de três municípios com diferentes rendas domiciliares per capita. Estudos Interdisciplinares sobre o Envelhecimento, v. 21, n. 1, p. 217-233, 2016. Disponível http://www.seer.ufrgs.br/index.php/RevEnvelhecer/article/view/59644/40725

em:

LEVORATO, C. D., MELLO, L. M., SILVA, A. S., NUNES, A. A. Fatores associados à procura por serviços de saúde numa perspectiva relacional de gênero. Ciência \& Saúde Coletiva, v. 19, n. 4, p. 12643-1274, $2014 . \quad$ DOI: http://dx.doi.org/10.1590/1413-81232014194.01242013.

LUZ, F. E.; SANTOS, B. R. M.; SABINO, W. Estudo comparativo de mortalidade por doenças cardiovasculares em São Caetano do Sul (SP), Brasil, no período de 1980 a 2010. Ciência \& Saúde Coletiva, v. 22, n. 1, p. 161-168, 2017. DOI: http://dx.doi.org/10.1590/1413-81232017221.18362015

MALLMANN, D. G.; GALINDO NETO, N. M.; SOUSA, J. C.; VASCONCELOS, E. M. R. Educação em saúde como principal alternativa para promover a saúde do idoso. Ciência \& Saúde Coletiva, v. 20, n. 6, p. 1763-1772, 2015. DOI: http://dx.doi.org/10.1590/1413-81232015206.02382014 
MANSUR, A. P.; FAVARATO, D. Mortalidade por doenças cardiovasculares no Brasil e na região metropolitana de São Paulo: atualização 2011. Arquivos Brasileiro de Cardiologia, v. 99, n. 2, p. 755-761, 2012. DOI: http://dx.doi.org/10.1590/S0066$782 \times 2012005000061$

MARQUES, A. P.; MONTILLA, D. E. R.; ALMEIDA, W. S.; ANDRADE, C. L. T. Internação de idosos por condições sensíveis à atenção primária à saúde. Hospitalization of older adults due to ambulatory care sensitive conditions. Revista de Saúde Pública, v. 48, n. 5, p. 817-826, $2014 . \quad$ DOI: http://dx.doi.org/10.1590/S0034-8910.2014048005133

PAGOTTO, V.; SILVEIRA, E. A.; VELASCO, W. D. Perfil das hospitalizações e fatores associados em idosos usuários do SUS. Ciência \& Saúde Coletiva, v. 18, n. 10, p. 3061-3017, 2013. DOI: http://dx.doi.org/10.1590/S1413-81232013001000031

SILVA, J. A.; HANSEL, C. G.; SILVA, J. Quality of life from the perspective of older adults with cancer: primary care nursing implications. Revista Enfermagem UERJ, v. 24, n. 3, p. e9621, 2016. DOI: http://dx.doi.org/10.12957/reuerj.2016.9621 